\title{
Philosophiques
}

\section{Justice et théories contestées du territoire}

\section{Margaret Moore}

Volume 39, numéro 2, automne 2012

Territorialité, identité nationale et justice mondiale

URI : https://id.erudit.org/iderudit/1013689ar

DOI : https://doi.org/10.7202/1013689ar

Aller au sommaire du numéro

Éditeur(s)

Société de philosophie du Québec

ISSN

0316-2923 (imprimé)

1492-1391 (numérique)

Découvrir la revue

\section{Citer cette note}

Moore, M. (2012). Justice et théories contestées du territoire. Philosophiques, 39(2), 339-351. https://doi.org/10.7202/1013689ar

\section{Résumé de l'article}

Les questions de justice soulevées par la possession du territoire sont nombreuses. Qui a droit à quoi ? La distribution est-elle équitable ? Quels sont les droits censés découler d'un droit au territoire ? Et il y en a bien d'autres. Le présent article met en évidence que ces questions de justice sont abordées sous une perspective plutôt différente selon la conception que l'on se fait du territoire. Il existe à ce dernier égard deux courants dominants : le premier, souvent identifié à Locke, voit le territoire sous l'angle de la propriété ; le second, que l'on rattache à Kant, est considéré comme le domaine géographique du pouvoir juridictionnel.
Ce document est protégé par la loi sur le droit d'auteur. L'utilisation des services d'Érudit (y compris la reproduction) est assujettie à sa politique d'utilisation que vous pouvez consulter en ligne.

https://apropos.erudit.org/fr/usagers/politique-dutilisation/ 


\title{
Justice et théories contestées du territoire
}

\author{
MARGARET MOORE
}

\begin{abstract}
RÉSUMÉ. - Les questions de justice soulevées par la possession du territoire sont nombreuses. Qui a droit à quoi ? La distribution est-elle équitable? Quels sont les droits censés découler d'un droit au territoire? Et il y en a bien d'autres. Le présent article met en évidence que ces questions de justice sont abordées sous une perspective plutôt différente selon la conception que l'on se fait du territoire. Il existe à ce dernier égard deux courants dominants: le premier, souvent identifié à Locke, voit le territoire sous l'angle de la propriété; le second, que l'on rattache à Kant, est considéré comme le domaine géographique du pouvoir juridictionnel.
\end{abstract}

\begin{abstract}
There are many justice issues raised by the possession of territory; questions of who is entitled to what; the fairness of the distribution; and the entitlements that are thought to follow from having a right to territory, to name a few. This paper then goes on to show that these justice issues are framed somewhat differently depending on one's conception of territory. There are two dominant conceptions of territory: territory as property; and territory as the geographical domain of jurisdictional authority. The former is often identified with Locke, and the latter with Kant.
\end{abstract}

Cet article porte sur la relation entre justice et territoire. Les théories de la justice devraient s'intéresser au territoire et aux droits territoriaux, puisque de nombreuses questions de justice très importantes s'inscrivent dans l'idée qu'un peuple détient des droits sur un territoire et que diverses conceptions de ce dernier intègrent les dimensions de justice à différents titres. Quiconque s'intéresse au territoire devrait également prêter attention à la justice, puisque les divers "droits" qui sont souvent associés à l'idée de droit territorial sont considérés comme étant limités par des considérations d'ordre juridique. Dans le présent article, je vais souligner certaines des façons dont justice et territoire s'entrecroisent, puis traiter brièvement de la manière dont les différentes théories du territoire conceptualisent les questions de justice en jeu.

Les questions liées au territoire et aux droits territoriaux ont récemment reçu une certaine attention philosophique ${ }^{1}$, mais elles sont toujours relativement sous-théorisées, et les questions complexes concernant le territoire n'ont pas retrouvé leur place dans l'étude d'autres problématiques sur

1. Voir David Miller, On Nationality, Oxford, Oxford University Press, I995; Tamar Meisels, Territorial Right, 2nd edition, Dordrecht, Springer, 2009; Avery Kolers, Land, Conflict and Justice; a political theory of Territory, Cambridge, Cambridge University Press, 2009; Margaret Moore, Ethics of Nationalism, Oxford, Oxford University Press, 200 I ; Annie Stilz, "Nations, States and Territory", Ethics, vol. I2I (avril 20II), p. 572-60I. 
lesquelles elles s'appuient. Le manque d'intérêt relatif pour le territoire est typique de la plupart des théories de relations internationales, qui présument qu'avoir des droits sur le territoire participe de ce que signifie être "souverain "; la souveraineté, particulièrement celle d'un État, est présupposée relever d'un domaine géographique. En effet, le fait de posséder le contrôle sur un territoire, ou des droits territoriaux, est souvent considéré comme définissant ce qu'est un État ${ }^{2}$. Par exemple, en vertu de la Convention de Montevideo sur les droits et devoirs des États (article premier) de 1933, un «État en tant qu'entité du droit international doit posséder les éléments suivants: une population permanente, un territoire défini, un gouvernement, et la capacité d'entrer en relation directe avec les autres États ${ }^{3}$ ».

Même en théorie politique, les domaines territorial ou géographique de la «souveraineté » ou de l'«État» n’ont pas été analysés séparément ni même été perçus comme soulevant des questions particulières, jusqu'à très récemment. À cet égard, la façon dont Rawls considère la justice et l'État dans Théorie de la justice est exemplaire: il commence avec l'hypothèse commodément simplificatrice que la société juste est fermée, qu'il s'agit d'une «association, plus ou moins autosuffisante, de personnes ${ }^{4}$ », faisant ainsi abstraction de la question du territoire auquel a droit «la société juste» et de la relation de ce territoire avec d'autres territoires. La conception de Rawls selon laquelle la justice est fonction des principes qui devraient gouverner la structure institutionnelle de base de la société, qu'il a, à des fins de simplification, conceptualisée comme une entité autosuffisante, a provoqué un débat considérable parmi les personnes qui s'intéressent à la justice mondiale. Les théoriciens de la justice mondiale se sont inscrits en faux contre Rawls pour ce qui est de limiter la portée de la justice à l'État, insistant sur le fait que l'espace mondial n'est pas simplement une arène où s'appliquent les restrictions de moralité ainsi que les règles de justice ${ }^{5}$, et ils ont élaboré des arguments justificatifs distincts à l'appui de cette prise de position ${ }^{6}$.

2. A. John Simmons, «On the Territorial Rights of States », Philosophical Issues: Social, Political and Legal Philosophy, vol. I I (200I), p. 32I.

3. Simmons, "On the Territorial Rights of States", p. 32I, note 5; en français, http:// omnilogie.fr/O/Le_principe_des_micronations_ou_le_d \% C3 \% A9sir_ultime_d'autonomie (consulté le 8 juin 20I2).

4. John Rawls, A Theory of Justice, Cambridge, Mass., Harvard University Press, I97 I, p. 4 ; en français, Théorie de la justice, Paris, Points, 2009, p. 30, traduction Catherine Audard.

5. Gerry Cohen et Liam Murphy rejettent la division entre justice et moralité, institutions sociales et moralité personnelle. Liam Murphy, «Institutions and the Demands of Justice", Philosophy \& Public Affairs, vol. 27 (I999), p. 25I-29I, à la p. 280; et G. A. Cohen, "Where the Action Is: On the Site of Distributive Justice ", Philosophy \& Public Affairs, vol. 26 (I997), p. 3-30, aux p. 22-23.

6. Tant Thomas Pogge dans World Poverty and Human Rights, Londres, Polity Press, 2002, que Charles Beitz dans Political Theory and International Relations, Princeton, Princeton University Press, I979, préconisent une forme d'institutionnalisme, c'est-à-dire qu'ils adhèrent au point de vue de Rawls, selon lequel la justice s'applique à un ensemble d'institu- 
Toutefois, le fait que Rawls présume également que la justice déborde les frontières des territoires n'a que peu retenu l'attention, et son analyse contient des suppositions importantes, implicites mais contestables, des rapports entre l'État, le peuple et le territoire.

La présomption générale de la plupart des théories politiques contemporaines a souvent tourné autour de l'idée que ce qui justifie les États, peu importe ce que c'est (par ex., la production de valeurs communes, telles que la paix et l'ordre), et quelle que soit sa nature, justifie également le territoire possédé par les États et les diverses dimensions de la souveraineté de l'État. Les théoriciens de la justice mondiale ont éprouvé un certain scepticisme à propos des arguments étatistes, mais ils prônent rarement le cosmopolitisme politique, aussi ont-ils également besoin de traiter des questions de territoire et des différentes questions juridiques qui sont ainsi soulevées.

Les droits au territoire sont habituellement compris comme étant configurés de façon analogue aux droits de propriété, soit un mélange complexe de droits à la revendication, de pouvoirs, de libertés et d'immunités ${ }^{7}$. Ces droits s'étendent habituellement sur quatre dimensions différentes. Premièrement, c'est une question de droit à l'autorité juridictionnelle, ce qui signifie un droit de légiférer et de faire appliquer des lois dans le domaine géographique. Deuxièmement, il existe des droits pour contrôler ou interdire tout mouvement à travers les frontières du territoire. Troisièmement, il y a un droit de taxer, réglementer et contrôler les ressources naturelles à l'intérieur du territoire de l'État ${ }^{8}$. Quatrièmement, bien qu'il ne fasse généralement pas partie des dimensions du droit territorial, il existe un droit à la défense nationale. Ce dernier est un droit sur le territoire, puisque les États sont censés être habilités à s'engager dans des guerres de défense en cas d'attaque de leur territoire. L'argument principal en faveur d'un droit défensif - décrit dans les textes comme étant l'argument de l'autodéfense - relève du droit à l'autodéfense individuelle, mais cela ne semble pas pertinent, puisque l'agression territoriale est habituellement considérée comme introduisant un droit à l'autodéfense, même dans les cas où personne n'est blessé, et même si le territoire n'est pas habité par qui que ce soit ${ }^{9}$. Une certaine définition de la terre ou du territoire est donc nécessaire pour justifier cet

tions, mais ils affirment que, en cette époque de mondialisation croissante, l'ordre mondial constitue un ensemble d'institutions et que l'appartenance à des systèmes institutionnels est une question de pertinence morale.

7. Cela correspond à la typologie conçue par Wesley Newcomb Hohfeld, qui fait la distinction entre différents types de droits en fonction des rapports normatifs entre détenteurs de droits et porteurs d'obligations. Voir Wesley Newcomb Hohfeld, Fundamental Legal Conceptions as Applied in Judicial Reasoning, Arthur Corbin (dir.), Westport, Connecticut, Greenwood Press, I978; et Carl Wellman, A Theory of Rights: Persons under Laws, Institutions and Morals, Alanheld, New Jersey, Rowman and Littlefield, I98 5.

8. Simmons, "On the Territorial Rights of States", p. 306.

9. Voir David Rodin, War and Self-Defence, Oxford, Oxford University Press, 2005. 
élément d'une théorie de guerre juste. Selon David Miller, une théorie du territoire devrait offrir un argumentaire qui puisse expliquer de façon plausible et cohérente l'interrelation entre ces divers éléments du droit territorial ${ }^{10}$.

Elle devrait également faire partie d'une théorie globale de la justice, cela en partie parce que chacune des quatre dimensions du droit territorial soulève de nombreuses questions juridiques distinctes. En effet, la notion selon laquelle l'autorité territoriale possède ces droits présuppose que celleci constitue un ordre politique juste ou légitime. Le droit juridictionnel, par exemple, soulève des questions à propos de la portée de l'autorité juridictionnelle et des limites de cette autorité (particulièrement les limites mises en place par la justice), ainsi que du traitement approprié des personnes qui sont touchées par les politiques et règlements appliqués, mais qui ne sont pas citoyens de l'État et, par conséquent, n'ont aucun moyen d'agir sur lesdites politiques. Les règlements concernant le mouvement des biens et des personnes à travers les frontières - et surtout ceux qui ont à voir avec l'immigration - soulèvent un grand nombre d'importantes questions de justice. L'une d'entre elles porte en premier sur l'équité même de tels règlements: certains peuvent soutenir que ceux-ci violent des droits fondamentaux de la personne, comme le droit au libre mouvement ${ }^{11}$. Même si nous ne soutenons pas ce point de vue, les lois elles-mêmes ont d'importantes conséquences distributives, tant dans les sociétés hôtes que dans les sociétés d'origine. Le contrôle sur les ressources naturelles est un des éléments les plus contestés du droit territorial, puisque les pays ne sont pas tous dotés de façon égale; il semble injuste que certains peuples puissent tirer profit d'une région fertile, riche en ressources et en ports naturels, alors que d'autres sont confinés à des régions relativement pauvres, sans ressource et enclavées. Et enfin, la question de la guerre juste est l'une des plus problématiques, puisque, en temps de guerre, de nombreuses personnes sont tuées et que les droits à la défense nationale (qui ne peuvent, selon la formule traditionnelle, découler que d'une attaque sur le territoire) comprennent un droit de tuer des combattants, ce qui, du moins en premier lieu, semble être en violation de leur droit à la vie. Il est donc profondément problématique que ce droit soit fondé sur une attaque (sans effusion de sang) d'un territoire seulement, et nous avons besoin, pour justifier cela en termes de démocratie libérale, d'une bonne théorie sur ce qu'est le territoire et sur les types de valeurs morales qui y sont enracinées. De nombreuses considérations juridiques importantes sont donc soulevées par les différentes dimensions du droit territorial, et il

10. David Miller, «Territorial Rights: Concept and Justification", Political Studies, vol. 60, no 2 (20I2), p. $252-268$.

11. Joseph Carens, "Open Borders and the Claims of Community ", conférence donnée lors d'une rencontre de l'American Political Science Association, à Toronto, en Ontario, du 2 au 5 septembre 2009 . 
est essentiel de les analyser, non seulement comme des politiques qui ont des incidences particulières sur les peuples, mais comme des dimensions d'un contrôle sur le territoire.

Dans le présent article, je ne tente pas d'analyser toutes les questions relatives à la justice qui découlent des différents aspects du droit territorial, ni de comparer ceux-ci aux effets des diverses théories du territoire sur les façons dont la notion de justice est encadrée. J'essaie, plus modestement, de souligner les argumentaires principaux concernant la nature même du territoire et de présenter quelques-unes des façons dont ceux-ci influent sur ce que nous estimons constituer une question de justice.

\section{Conceptions différentes du territoire}

Il existe, en gros, deux façons différentes de définir le territoire: le territoire en tant que propriété, et le territoire en tant qu'autorité juridictionnelle. Je vais ici présenter brièvement les différents argumentaires et leur relation générale avec des considérations de justice.

\subsection{Le territoire en tant que propriété}

L'un des raisonnements les plus développés est fondé sur la définition de la propriété individuelle de Locke, en vertu de laquelle le territoire est vu comme découlant d'un regroupement de possessions de propriété privée individuelle. À l'origine, la propriété est définie en général comme étant un droit individuel (naturel), et on conçoit le territoire comme étant généré par le consentement de nombreux possesseurs de propriété individuelle à mettre en place un gouvernement (législatif, exécutif et judiciaire) qui les dirige ${ }^{12}$. Dans le Second Traité, Locke nous demande d'imaginer que des personnes (individus ou personnes associées au sens large [par ex., les familles]), dont un grand nombre ont un titre de propriété sur une terre, s'associent pour créer un État. Comme l'écrit Simmons: "Quand les hommes consentent à fonder une société politique ou à se joindre à elle, leur consentement devrait être compris comme un consentement à tous les arrangements qui sont

12. Dans le Second Traité, Locke affirme qu'il existe un droit naturel à la propriété privée, découlant de l'idée que tous les hommes ont un droit sur les fruits de la terre. Ce droit fondamental (à la subsistance) se combine, selon Locke, avec la notion que le travail est le moyen par lequel les individus peuvent transformer en propriété privée des objets extérieurs de l'état de nature. Le droit à la propriété privée est limité par le droit à la subsistance, ou plus précisément, par le besoin que les hommes ne puissent s'approprier légitimement des terres que "s'il reste aux autres assez de semblables et d'aussi bonnes choses communes ", bien que cet argument plutôt fort de la clause soit par la suite grandement atténué. John Locke, The Second Treatise of Government, Thomas P. Peardon (dir.), Indianapolis, Bobs-Merill Educational Publishing, I952, [publié à l'origine en I690], chap. V, p. I6; en français, Traité du gouvernement civil, traduction de David Mazel en $\mathrm{I} 795$ réalisée à partir de la 5 édition de Londres publiée en I728 (http://classiques.uqac.ca/classiques/locke_john/traite_du_gouvernement/traite_ du_gouv_civil.doc, consulté le 8 juin 20I2). 
nécessaires à une société stable et paisible ${ }^{13}$.» Locke affirme que le peuple consentirait au gouvernement de la majorité, à lui obéir et à soutenir les lois (dans les limites de la loi naturelle) et, fait important pour cet argumentaire sur le territoire, consentirait à intégrer les propriétés foncières légitimes dans ce territoire sur lequel la société aura juridiction ${ }^{14}$. Ainsi, la création du territoire est fonction du consentement des détenteurs individuels de la propriété, et le droit naturel à la propriété est, conceptuellement parlant, préalable à l'État et le socle de la juridiction de celui-ci. Le droit territorial légitime est établi, selon ce raisonnement, à travers la sujétion, librement consentie, des personnes et de leurs terres à l'autorité de l'État.

Un important avantage de cet argumentaire est qu'il explique facilement le lien entre les trois éléments qui sont normalement réputés faire partie du «droit territorial». Par exemple, si l'on considère que le territoire est semblable à la propriété, il sera plutôt aisé de soutenir qu'il existe une capacité d'en contrôler les frontières. Tout comme les propriétaires ont le droit d'empêcher les gens de pénétrer dans leur propriété, ils peuvent aussi collectivement restreindre l'accès d'autrui à leur État. Cela explique la troisième dimension du droit territorial, à savoir le droit aux ressources: cellesci appartiennent d'emblée aux individus, en tant que membres d'une union de propriétés foncières (déjà légitimes en droit). Et on peut ainsi expliquer légalement le droit à protéger le territoire, au moins dans sa dimension d'analogie avec le droit des individus à l'autoprotection de leur personne et de leurs biens.

Cet argumentaire individualiste lockien pose toutefois deux problèmes de taille, l'un comme l'autre liés à la transition entre propriétés foncières privées individuelles, et juridiction territoriale. Il ne s'agit pas de problèmes ayant trait au raisonnement sur la justice, mais plutôt relatifs à une conception du territoire en tant qu'union de propriétés individuelles. Selon les versions les plus élaborées de cette théorie (Simmons, Steiner), les individus qui détiennent de plein droit une propriété sur la terre acceptent l'établissement d'un gouvernement dans le but de préserver la paix, et pour faire respecter la loi naturelle. Une des difficultés les plus sérieuses ici est que le modèle de consentement de l'individu ne semble pas justifier l'autorité juridictionnelle contiguë des États territoriaux, où le caractère exécutoire de la loi s'applique à toute personne vivant à l'intérieur des frontières, alors qu'aucune terre sans propriétaire ou sous un autre statut n'existe à l'intérieur du territoire. Il semble ainsi peu probable que cet argumentaire puisse justifier le droit territorial tel que nous le connaissons, alors que droits territoriaux et en particulier autorité juridictionnelle s'appliquent uniformément et systématiquement à travers le territoire. Un autre problème soulevé par la perspective lockienne est qu'aucun État n'est dans les faits légitimé par le consentement

13. Simmons, "On the Territorial Rights of States", p. 3 I3.

14. Ibid., p. 3 I 4 . 
individuel des propriétaires fonciers, ce qui signifierait que tout État territorial existant est voué à l'illégitimité. Simmons, un anarchiste philosophique, se satisfait de cette conclusion: il pense que tous les États sont jusqu'à un certain point illégitimes, parce qu'ils ne sont pas véritablement fondés sur le consentement des individus libres à la juridiction de l'État ${ }^{15}$. Néanmoins, l'argument a peu de chance de persuader les non-anarchistes parmi nous qui pensent que les États peuvent être légitimes, et que le droit territorial a une existence bien réelle.

Une perspective dérivée de celle de Locke insiste sur les analogies étroites entre territoire et propriété, mais fait valoir que le territoire n'est pas le produit du consentement de propriétaires fonciers individuels dans l'exercice de leur droit naturel à la propriété. Plutôt, selon ce point de vue, l’État est un collectif qui peut détenir la propriété: le territoire de l'État est conçu de façon plus appropriée comme analogue à la propriété de l'État. Cette vision du territoire comme propriété détenue par un acteur collectif s'est sans conteste avérée extrêmement pertinente dans l'histoire ${ }^{16}$, et s'applique encore aux vastes étendues des fonds marins, ou encore aux régions désertes de l'Arctique et de l'Antarctique, sur lesquelles les États formulent les revendications les plus larges possibles dans le but d'en exploiter les ressources, comme le ferait un propriétaire foncier cherchant à tirer le meilleur profit de son bien ${ }^{17}$. Toutefois, ce point de vue ne semble pas pouvoir être appliqué globalement, parce qu'il ne peut expliquer le fait que le territoire est aussi également compris comme appartenant à tout le peuple, pour l'avantage de ce dernier. En effet, selon l'argumentaire du territoire en tant que propriété collective, la terre ne peut pas que faire l'objet d'une acquisition, elle doit également pouvoir être abandonnée; dans les faits, toutefois, il n'est pas

15. Simmons, "On the Territorial Rights of States", p. 3 I 6 .

16. Par le passé, le roi/la reine ou le souverain/la souveraine était lié(e) à l'entité juridictionnelle sur laquelle il/elle régnait d'une manière passablement semblable à la relation existant entre un/une propriétaire et son bien foncier: il/elle contrôlait (pour l'essentiel) cette entité et la transmettait par héritage, tout comme le faisaient les propriétaires fonciers. Le territoire juridictionnel du souverain/de la souveraine était apporté lors du mariage, comme une propriété l'aurait été. Beaucoup de mariages dynastiques étaient conclus pour élargir ou renforcer le royaume, et les mariages aristocratiques souvent décidés en fonction des terres que chacun des époux amenait dans la corbeille. Nombreuses sont les frontières actuelles des États européens qui ont été tracées de la sorte: l'Espagne contemporaine est dans une large mesure le fruit du mariage de Ferdinand d'Aragon et d'Isabelle de Castille. L'union de l'Angleterre et de l'Écosse a été facilitée par l'accession de James VI, roi d'Écosse, au trône d'Angleterre.

17. L'actuelle ruée vers les océans illustre bien que le territoire est une forme de propriété. Toutes les revendications d'un droit territorial sur les fonds marins sont traitées par la Commission des limites du plateau continental, puis éventuellement renvoyées devant la Cour internationale de Justice à La Haye ou un tribunal spécial. Les revendications concurrentes sont réglées sur une base scientifique, par l'analyse des formations géologiques du plateau continental, et d'autres principes secondaires relatifs à la profondeur de l'océan et à la proximité de la terre. Cependant, les pays qui expriment une revendication semblent surtout voir les fonds océaniques comme une source potentielle de richesses ou une forme de propriété. 
clair que l'État puisse se départir de portions de territoire (avant-postes) qu'il ne veut plus détenir (comme les îles Falkland dans le cas du RoyaumeUni), surtout si la majorité de la population dudit avant-poste désire faire partie de cette juridiction politique (le Royaume-Uni).

Il existe deux définitions du territoire, complexes sur le plan théorique, toutes deux inspirées par Locke et sous-tendant le présent article, selon lesquelles il est propriété d'un sujet collectif. Dans son livre Territorial Right, Tamar Meisels élabore une théorie du territoire complexe et multicritères, mais dont l'argument central est que les nations sont propriétaires de la terre qu'elles occupent. Sa théorie est nationaliste dans la mesure où elle pense que les nations (et non l'État) possèdent des droits de propriété, définis comme un droit particulier (un droit qui naît d'une relation ou d'une transaction particulière). Selon cet argumentaire, en accomplissant des actions particulières en rapport avec le territoire, la nation crée un droit «naturel» fort eu égard à l'accès aux ressources qu'il renferme. À l'instar de Locke, Meisels pense que c'est le travail de la terre et sa transformation qui donnent à la nation un droit particulier sur elle. Comme dans la théorie de la propriété individualiste étudiée plus haut, il rapproche territoire et propriété et met l'accent sur la nature transactionnelle semblable du travail en retirant son état de nature à la terre et en transformant celle-ci en territoire d'un groupe particulier. Mais, à la différence des argumentaires antérieurs de Simmons et Steiner sur la propriété individualiste, celui de Meisels introduit une approche collectiviste du territoire en tant que propriété: pour lui, c'est un groupe d'individus (une nation) qui peut obtenir des droits territoriaux sur certaines terres par son travail collectif sur celles-ci.

Dans le droit fil de Meisels, Cara Nine propose une théorie du droit au territoire à l'approche lockienne assumée. Selon son raisonnement, toutefois, ce n'est pas la nation qui obtient les droits territoriaux, mais l'État; Nine écrit: "Selon [ma] [...] théorie lockienne collectiviste, l'État acquiert des droits territoriaux essentiellement de la même façon que les individus acquièrent des droits de propriété (c'est moi qui souligne) ${ }^{18}$.» Elle poursuit:

L'État modifie la terre de la même manière que les individus par le travail. Les lois et coutumes régissant l'utilisation de la terre créent des systèmes uniques d'agriculture, et d'autres formes d'utilisation des ressources à plusieurs niveaux: I) les systèmes de marché créés et gérés par les institutions politiques déterminent et génèrent de la valeur pour les terres elles-mêmes et pour tous les produits qui en sont issus. Les systèmes de marché influencent également la façon dont les ressources intérieures sont exploitées; 2) les individus, sous l'autorité des institutions politiques, exploitent les terres et leurs ressources selon certains principes mis de l'avant par l'ordre politique; 3 ) les États sont responsables de l'interprétation et de l'application des droits de propriété indi-

18. Cara Nine, "A Lockean Theory of Property", Political Studies, 2008, vol. 56, p. I 48 à I 65 , en p. I 55 . 
viduelle, et ces droits de propriété sont partie prenante aux décisions sur la manière d'exploiter les terres et sur ceux qui les exploitent. En créant, arbitrant et appliquant des lois, les États ont une capacité de modifier le monde qui peut être à la source de leur droit à revendiquer un territoire ${ }^{19}$.

L'argumentaire de Nine aide à établir le droit historique au territoire: dans le cas où un État a mis en œuvre des activités créatrices de richesses - transformé les terres par ses activités institutionnelles, ses lois et règlements, façonné le paysage par le zonage, les règles en matière de propriété privée, etc. —, il est fondé à jouir de ces droits à perpétuité.

Cette logique, cependant, semble prêter le flanc à deux axes de critiques sérieux, quoiqu'assez différents. Un des problèmes est que l'idée de territoire en tant que sorte de droit dont les États pourraient se prévaloir en vertu de leurs activités est profondément conservatrice en cela qu'elle fait des États les porteurs des droits collectifs. Cette idée laisse de côté le fait que les États eux-mêmes peuvent avoir été créés par la force ou la conquête et qu'il existe d'autres titulaires de droits potentiels, ou d'autres conceptions d'une autorité politique pouvant également réaliser ces activités qui confèrent des droits. Le raisonnement est conservateur dans la mesure où il ne tient compte que des États existants, sans égard à la façon dont ils ont été créés, et qu'il semble incapable d'envisager que de tels droits puissent avoir été engendrés par d'autres agents. Il suppose que le régime existant doive être soutenu en toutes circonstances dans son action en faveur des activités de création de richesse (par comparaison avec une situation correspondante de non-État); mais en fait, l'État exclut d'autres prétendants potentiels par la coercition, même s'ils ont la volonté et la capacité de produire une législation, un zonage, etc. C'est un argument conservateur qui transfère le droit territorial à tous les États existants, mais qui n'explique pas véritablement le fondement originel de la légitimité de l'État ou la façon dont il en est venu initialement à exercer son autorité sur sa juridiction.

En résumé, le raisonnement selon lequel l'État est le premier titulaire des droits territoriaux est trop conservateur et trop minimaliste (dans ses normes fonctionnelles) pour être convaincant. Il convient d'approfondir la question relative au peuple vivant sur le territoire, au peuple pris collectivement au nom duquel l'État exerce son autorité, ce qui ne pourra que renforcer les liens d'un tel argumentaire avec la notion de justice. Le point de vue selon lequel le territoire de l'État se résume à sa propriété ou à un analogue de celle-ci ne passe pas le test de la légitimité que l'on reconnaît normalement à l'État et non aux propriétaires fonciers, qui sont libres de traiter bien ou mal leur propriété sans que cela ne soulève de questions quant à leur droit à cette dernière. Telle qu'elle s'exprime là, l'idée que l'État est un propriétaire foncier renvoie au seul État et à son territoire. Elle ne précise pas le

19. Cara Nine, «A Lockean Theory», p. I 55. 
lien qu'entretient avec lui le peuple qui vit en son sein, et ne dit pas non plus clairement en quoi l'État propriétaire foncier pourrait incarner d'une façon ou d'une autre l'idée d'un État garant d'un traitement équitable et du bon fonctionnement de la justice.

\section{Justice}

Cela m'amène à la théorie dominante, inspirée de Kant, selon laquelle la notion de territoire est définie par le domaine où s'exerce le pouvoir juridictionnel, ou la zone géographique dans laquelle un gouvernement autonome est à l'œuvre, où les droits sont respectés et la justice rendue. Cette vision est à l'exact opposé de celles que nous venons d'étudier, qui perçoivent le territoire comme une forme de propriété, dérivée des propriétés foncières individuelles ou d'un droit de propriété détenu par l'État. Selon la perspective d'un pouvoir juridictionnel, une telle interprétation est erronée: les droits de propriété sont non seulement distincts de la juridiction de l'État, mais ils en découlent. C'est l'État, dans l'exercice de son pouvoir juridictionnel, qui détermine les droits de propriété, par des règlements de zonage, des droits de legs et de transmission, et autres droits de propriété ${ }^{20}$.

Kant et les kantiens contemporains avancent que les individus ont l'obligation de quitter un État sans autorité politique et qui ne peut donc garantir la justice, et de s'en remettre à un pouvoir juridictionnel commun. Cette obligation trouve son fondement dans la possession de choses dans le monde extérieur (que l'on ne peut pourtant pas à proprement parler qualifier de "propriété»). D'un côté, cette possession est vue comme une extension de la liberté individuelle au sens où les personnes ont la possibilité de choisir librement et d'utiliser des objets dans le monde extérieur pour satisfaire les aspirations qu'elles ont définies librement pour elles-mêmes. La liberté humaine suppose donc la possession ou l'acquisition d'objets extérieurs. Néanmoins, de prime abord, la propriété semble aussi entrer en contradiction avec l'idée de liberté, puisque le fait de soustraire des choses à l'usage commun dans le monde extérieur empêche d'autres personnes de jouir de la propriété et constitue une entrave inappropriée à leur libertée ${ }^{1}$. Cela contredit donc la doctrine kantienne dans la mesure où Kant affirme aussi que notre droit inné à la liberté doit «s'accorder avec la liberté de chacun suivant une loi générale ${ }^{22} »$. La seule voie de contournement de cette difficulté consiste à reconnaître la réciprocité des droits de tous à la pro-

20. On trouvera une réflexion intéressante sur un argumentaire nationaliste (non étatiste) du pouvoir juridictionnel dans «Territorial Rights: Concept and Justification », de David Miller, à paraître dans Political Studies.

21. Emmanuel Kant, «The Metaphysics of Morals», dans H. Reiss (dir.), Kant's Political Writings, Cambridge, Cambridge University Press, I970, p. I37-I38; Kant, Métaphysique des moeurs, Principes métaphysiques de la doctrine du droit, traduction française Alain Renaut, Paris, Garnier-Flammarion, 1994.

22. Kant, Éléments métaphysiques de la doctrine du droit..., p. 44. 
priété, à travers la création d'une autorité juridictionnelle. Puisque l'exercice de ma liberté semble obligatoirement aboutir à la restriction de celle d'autrui, le dilemme ne peut trouver sa résolution que par la reconnaissance réciproque de l'obligation de tous de respecter les principes fondamentaux entourant l'acquisition, la transmission et l'utilisation des objets dans le monde extérieur, ce que nous considérons normalement comme les «droits de propriété ». Cette reconnaissance réciproque exige la création d'une condition civile, qui assure que ma liberté est respectée, de même que celle d'autrui, une assurance qui ne peut exister dans l'état de nature. L'utilisation de choses extérieures dépend dès lors, non plus de la volonté coercitive d'une personne, mais de la volonté générale d'une autorité juridictionnelle, en proposant un cadre de règles générales, fondement de la société civile. Bien que la propriété joue un rôle important dans l'explication de la soumission au pouvoir juridictionnel, l'idée est que la création d'un domaine d'autorité juridictionnelle est essentielle pour être en phase avec l'impératif catégorique d'une liberté égale pour tous. L'élan cosmopolite de l'invocation kantienne du territoire comme domaine géographique de l'autorité juridictionnelle décrit le mouvement des individus d'un État de non-droit hypothétique vers une condition «justifiée », où le territoire de l'État est vu comme le domaine géographique de son propre pouvoir juridictionnel et où il est légitimé à appliquer le principe kantien du droit ${ }^{23}$. Il n'est toutefois pas très utile de décrire le principe de subsidiarité pour passer de la justification des droits territoriaux, rattachés aux États en général, pour expliquer comment des États particuliers peuvent détenir une autorité sur certains territoires particuliers ${ }^{24}$.

Alors que l'État est considéré d'une certaine façon comme appartenant à tout le peuple, et comme agissant au bénéfice de tous, il n'existe aucun principe normatif ou théorie particulière pour différencier les frontières qui délimitent les unités dans lesquelles la justice devrait être dispensée. De nombreux libéraux adoptent l'argument de Kant et justifient l'État en général, mais non un État particulier. Les frontières sont ou bien totalement sous-théorisées, ou bien, quand elles sont prises en considération, vues comme le résultat d'une conjoncture historique, d'une conquête ou d'une

23. L'argument de Kant selon lequel les pleins droits à la propriété dépendent de la mise en place d'une volonté omnilatérale est mieux présenté en termes de problèmes avec l'autorité juridictionnelle inhérente aux acteurs non étatiques. Comme le souligne Stilz dans sa discussion des droits territoriaux kantiens, l'exercice de la juridiction par des acteurs non étatiques est sujet à des problèmes d'interprétation et de coercition unilatérales. Annie Stilz, "Nations, States and Territory", Ethics, vol. I2I (avril 20II), p. 572-60I aux p. 58I-582.

24. Comme l'affirme Miller, ce raisonnement n'est pas clair pour ce qui est de définir la façon dont peuple(s) et territoires particuliers sont liés. Il illustre son propos en faisant remarquer que cette théorie n'explique pas ce qu'un individu devrait faire lorsqu'il est entre deux États différents, chacun revendiquant le pouvoir juridictionnel, chacun promettant de garantir les droits de propriété par l'application du cadre de droit public de l'État. David Miller, "Property and Territory: Locke, Kant, and Steiner ", Journal of Political Philosophy, vol. I9, ${ }^{\circ}$ I (20II), p. 90-IO9 à la p. I04. 
dynamique politique, mais pas comme étant sujettes à des considérations de justice. Sur cette base, de nombreuses théories libérales de la justice (axées sur l'État) peinent à soulever les questions de frontières, et tendent à traiter de celles-ci comme d'une simple donnée empirique, extérieure à la théorie de la justice. De nombreux libéraux, pour des raisons de pragmatisme, voient dans les frontières une base à partir de laquelle la théorie s'échafaude, le cosmopolitisme politique n'étant pas à l'ordre du jour dans un futur prévisible. Le problème de cette approche est que si l'État est perçu comme un lieu de justice, et comme nécessaire à la réalisation de la justice (le droit), le domaine territorial de l'État, lui, n'est pas théorisé clairement en termes de justice ou, s'il l'est, le fait que les territoires soient rarement égaux est vu comme un problème de justice, puisqu'il est évident que certains territoires sont plus vastes et fertiles, ou plus riches en ressources que d'autres.

Outre les limites inhérentes à un argumentaire du droit territorial fondé sur la justice lorsque l'on individualise le territoire, ou que l'on «attribue » des territoires particuliers à des groupes ou États particuliers, l'argumentaire fondé sur la justice tend à ne pas justifier les États, pour ce qui est de leurs droits sur le territoire, d'une manière conforme à notre compréhension habituelle du droit territorial. Bien que certains n'y voient pas un problème pour la théorie, il n'est pas sans intérêt de noter que le raisonnement mettant l'accent sur la justice propre au droit territorial semble n'octroyer ce dernier que dans la mesure où les États satisfont aux normes requises de justice. Mais, comme il s'avère, de nombreux États échouent en cette matière: il peut s'agir d'États en déroute ou d'une certaine façon illégitimes, ou peutêtre simplement que les normes de justice auxquelles ils se conforment ne sont pas à la hauteur de celles d'un État voisin, concurrent. Il semble qu'ils pourraient donc perdre leur droit territorial, et qu'un État plus juste et efficace mériterait par là même ce droit (le contrôle sur les ressources, les frontières, etc.). Cela tend à dire que l'argument de la justice pourrait être efficace dans la justification de tels droits, mais qu'elle ne l'est pas autant en matière de droit territorial tel qu'on le conçoit habituellement en droit international. En effet, l'argumentaire est sous-tendu par des incidences révisionnistes. Fait intéressant, la plupart de ceux qui adhèrent à ce raisonnement tendent, placés devant ces incidences, à adopter un discours de légitimité minimal et suggèrent qu'un État le moindrement juste devrait voir son territoire protégé. Tom Christiano, par exemple, s'en prévaut dans son invocation d'un "principe de conservation ${ }^{25}$ ", de même qu'Allen Buchanan, qui affirme que dans un État légitime (notion plutôt différente de celle d'un État totalement impartial) l'intégrité territoriale devrait être protégée. Cependant, il n'est

25. Tom Christiano, «A Democratic theory of Territory and Some Puzzles about Global Democracy ", Journal of Social Philosophy, vol. 37, n I (2006), p. 8 I-I07; Allen Buchanan, Justice, Legitimacy, and Self-determination: Moral Foundations for International Law, New York, Oxford University Press, 2004. 
pas sûr que ce raisonnement soit tout à fait en phase avec l'argumentaire fondé sur la justice qu'ils mettent de l'avant, celui-ci semblant montrer une préférence ad hoc pour le statu quo plutôt qu'une position découlant des principes fondés sur la justice que tous deux embrassent.

\section{Condusion}

Dans cet article, j'ai présenté les deux courants dominants en matière d'argumentaire sur le territoire, du point de vue de leurs défenseurs contemporains: le premier, dérivé de Locke, le deuxième, de Kant; j'ai également souligné dans les deux cas une difficulté à produire un argumentaire concernant certains éléments que nous associons normalement au "droit territorial». Pour ces deux écoles, la justice est importante. Dans l'argumentaire lockien, la propriété est un droit naturel, et le territoire est à bon droit propriété du détenteur de droit (territorial), qu'il s'agisse des propriétaires individuels pris collectivement, ou de l'État en tant que collectivité. Ce raisonnement explique la démarcation des territoires individuels et constitue en lui-même un argument de justice (procédurale) puisqu'il est fondé sur une théorie de la propriété à bon droit. Le point de vue kantien met la justice au centre de la justification de l'État dans l'exercice de ses pouvoirs, mais son domaine territorial, qui devrait lui-même soulever des questions sur la distribution équitable d'avantages et de responsabilités, n'est pas théorisé comme une question de justice, ou est perçu curieusement comme une injustice évidente, les territoires étant rarement exactement semblables et jamais découpés de manière équivalente. Enfin, cet argumentaire semble avoir des incidences révisionnistes dans le monde actuel (plutôt injuste), mais les théoriciens se réclamant de cette école tendent à les balayer en adoptant curieusement un discours de justice ou de légitimité minimales. 